\title{
High frequency oscillations in limbic rat model for temporal lobe epilepsy
}

Address: ${ }^{1}$ Department of Biomedical Engineering, University of Florida, Gainesville, FL 32611, USA, ${ }^{2}$ Department of Neuroscience, University of Florida, Gainesville, FL 32611, USA, ${ }^{3}$ Department of Pediatrics, University of Florida, Gainesville, FL 32611, USA and ${ }^{4}$ Department of Neurology, University of Florida, Gainesville, FL 32611, USA

Email: Dong-Uk Hwang* - dhwang@bme.ufl.edu

* Corresponding author

from Sixteenth Annual Computational Neuroscience Meeting: CNS*2007

Toronto, Canada. 7-12 July 2007

Published: 6 July 2007

BMC Neuroscience 2007, 8(Suppl 2):PI45 doi:I0.I I86/I47|-2202-8-S2-PI45

(C) 2007 Talathi et al; licensee BioMed Central Ltd.

Recently a number of groups $[1,2]$ have reported on the existence of pathological High frequency oscillations (HFO's) (oscillations in the frequency range of 80-200 $\mathrm{Hz}$, termed as Ripple band and oscillations in the frequency range of $200 \mathrm{~Hz}$ and above, termed as Fast Ripple band) in the epileptic brain both in in-vivo and in-vitro experiments. Our goal in this study is to study the statistical modulation of HFOs during epileptogenesis in order to characterize their function in progression to seizures in the epileptic brain.

In this study we define a HFO event as a subset of wave having significant high frequency component with low wave amplitude. HFO are detected from data recorded at a sampling rate of $12000 \mathrm{~Hz}$ for the entire duration of epileptogenesis which lasts anywhere from about 3-6 weeks.

Statistical analysis on the HFO suggest that occurrence of HFO's occur primarily during the 12 hour dark cycle whereas the HFO's primarily seem to occur during the 12 hour day cycle in the control rat The video recording shows that the rat is primarily in active and exploratory state during the dark cycle. These observations suggest that HFO in epileptic rats are correlated with the state of arousal.
Spatial correlation of HFOs in different regions of the brain is also investigated with cross-correlogram. Comparison of cross-correlogram of the post-stimulus HFO in the epileptic rat to the pre stimulus HFO (control) suggests modification in the circuitry in the hippocampus, evidence for which in in-vitro experiments were provided by [3].

\section{References}

I. Engel J Jr, Wilson CL, Bragin A: Advances in understanding the process of epileptogenesis based on patient material: what can the patient tell us? Epilepsia 2003, 44(suppl I2):60-7I.

2. Bragin A, Engel J, Wilson CL, Fried I, Buzsaki G: High frequency oscillations in human brain. Hippocampus 1999, 9:137-142.

3. Avoli M, D'Antuono M, Louvel J, Kohling R, Biagini G, Pumain R, D'Arcangelo G, Tancredi $V$ : Network and pharmacological mechanisms leading to epileptiform synchronization in the limbic system in vitro. Prog Neurobiol 2002, 68:167-207. 